\title{
Management, risk factors and outcome of cranial dural arteriovenous fistulae: a single-center experience
}

\author{
Stephan Dützmann • Jürgen Beck • Rüdiger Gerlach • Andrea Bink • \\ Joachim Berkefeld • Richard du Mesnil de Rochement • Volker Seifert • \\ Andreas Raabe
}

Received: 17 November 2010 / Accepted: 17 February 2011/Published online: 20 March 2011

(C) Springer-Verlag 2011

\begin{abstract}
Background The role of endovascular interventions in managing dural arteriovenous fistulas (DAVFs) is increasing. Furthermore, in patients with aggressive DAVFs, different surgical interventions are required for complete obliteration or disconnection. Our objective was to evaluate the management of patients with intracranial DAVFs treated in our institution to identify the parameters that may help guide the long-term management of these lesions.

Methods The hospital records of 53 patients with intracranial DAVFs were reviewed. We then conducted a systematic telephone interview to obtain long-term follow-up information.

Results The main presenting symptoms were tinnitus and headache. Nineteen $(35 \%)$ patients presented with intracranial bleeding, $84 \%$ of patients scored between 0 and 2 using a modified Rankin Scale at the last follow-up visit. Twentyfour patients were treated surgically. Overall postoperative complications occurred in seven (29\%) surgically treated patients, but only two patients permanently worsened. For
\end{abstract}

Presentation at a conference This work has not been previously published in whole or in part.

S. Dützmann · J. Beck · R. Gerlach • V. Seifert · A. Raabe Department of Neurosurgery, Neurocenter, Johann Wolfgang Goethe University,

Frankfurt am Main, Germany

A. Bink $\cdot$ J. Berkefeld $\cdot$ R. du Mesnil de Rochement Department of Neuroradiology, Neurocenter, Johann Wolfgang Goethe University,

Frankfurt am Main, Germany

\section{A. Raabe $(\bowtie)$}

University Department of Neurosurgery,

Bern University Hospital, Inselspital,

3010 Bern, Switzerland

e-mail: susan.wieting@insel.ch patients with Borden type II and III fistulas, the annual incidence of hemorrhage was $30 \%$. Two patients had late recurrences of surgically and endovascularly occluded DAVFs. Long-term follow-up showed that compared with spinal DAVFs, only $50 \%$ of intracranial DAVFs showed complete remission of symptoms, $41 \%$ partial remission, $6 \%$ no remission and $4 \%$ deterioration of symptoms that led to treatment of the DAVF.

Conclusion In general, intracranial DAVFs can be successfully surgically managed by simple venous disconnection in many cases. However, half of the patients do not show complete remission of symptoms. Age and the occurrence of perioperative complication were the most important determinants of outcome.

Keywords Dural arteriovenous fistula Endovascular treatment · Surgical treatment results $\cdot$ Intracranial hemorrhage

\section{Introduction}

Dural arteriovenous malformations, also called dural arteriovenous fistulas (DAVFs), are complex acquired vascular lesions. The classification systems that are widely accepted use venous drainage pattern to categorize DAVFs [3, 7]. DAVFs with retrograde leptomeningeal venous drainage or an associated venous varix are defined as high-risk fistulas and are associated with a grave natural history. Cortical venous reflux, either untreated or persistent after incomplete treatment, was reported to cause an annual mortality rate of $10.4 \%$ and an annual morbidity rate of $15 \%$ [36].

Hemorrhage or progressive neurological deficits compose the typical presentation of high-risk fistulae [17]. Rarely are they clinically quiescent. Cranial DAVFs without 
cortical venous drainage are considered to be benign, with a clinical presentation characterized by the absence of aggressive clinical events [30].

Prompt diagnosis and treatment of the aggressive lesions is mandatory. Primarily taking venous drainage into consideration, a careful evaluation is needed for the choice of treatment. Many DAVFs can be successfully managed with endovascular techniques [26]. However, a considerable number of patients require different forms of surgical treatment because they have DAVFs with anatomic features that are not suitable for endovascular treatment [17].

Studies reporting long-term results of treatment-either surgical or endovascular - are scarce. Here we systematically reviewed our experience with 53 patients with cranial DAVFs that were evaluated and treated at our institution. We were interested specifically (1) in the rate of events after diagnosis and before treatment, (2) after incomplete treatment, (3) after complete DAVF occlusion, and (4) the recurrence rate.

\section{Materials and methods}

First, we retrospectively reviewed the charts of our patients with intracranial DAVFs between 1999 and 2007. Patient records, cerebral angiograms, operative reports, and clinical notes were analyzed. We then conducted a systematic telephone interview to obtain long-term follow-up and to assess the modified Rankin Score (mRS) of the patient [21]. If the patient could not be reached via telephone or was not able to respond adequately, a close relative was contacted to provide the required information.

\section{Management}

All intracranial DAVFs were evaluated by an interdisciplinary team of neurosurgeons, neuroradiologists and neurologists. The assessment of patients included a detailed medical history, a full neurological examination, and at least one angiogram. In the angiogram the following features were evaluated: location of fistula, venous drainage and presence of retrograde leptomeningeal venous drainage. On the basis of proximity of involvement of the venous sinus, DAVFs were classified into the following locations: cavernous, transverse-sigmoid, superior sagittal sinus, ethmoidal, tentorial and jugular foramen DAVFs. We used the more neurosurgical oriented Borden classification of cranial DAVFs $[3,7]$.

Our team determined whether patients were candidates for potential endovascular cure or for preoperative embolization. If a Borden II or III fistula was not amenable to endovascular management (i.e., due to inaccessibility or diffuse arborization) or not cured by embolization, the patient underwent surgical treatment.

Intraoperatively, we used digital subtraction angiography (DSA) in selected cases to verify obliteration of the fistula. Intraoperative indocyanine green angiography (ICGA) was used after the advent of this technique in 2003 in our hospital. Postoperative imaging consisted of routine postoperative DSA performed between days $1-7$. We repeated the angiogram if the patient presented with new symptoms after a primary uneventful course. Routine follow-up angiography was not performed if a postoperative angiogram showed complete occlusion of the fistula.

\section{Statistical analysis}

Univariate associations were assessed by Pearson's $\chi^{2}$ test with continuity correction. Significance level was set at a $p$ value of less than 0.05. All statistical analyses were performed using the software program SPSS (SPSS, Chicago, IL, USA).

\section{Results}

Between 1999 and 2007 we treated 53 patients (male/ female ratio 1:1.08) at our institution (Fig. 1). The mean age at presentation was 57 years (range 25-61).

\section{Location}

DAVFs were situated in the transverse sigmoid sinus in 30 patients, in the superior sagittal sinus in 11 , and in the cavernous sinus in eight patients. Three patients had posterior fossa fistulas and one had an ethmoidal DAVF. All of the fistulas to the superior sagittal sinus, posterior fossa, and anterior cerebral fossa were Borden type III. Twelve Borden type II fistulas were found in the transversesigmoid sinus and five in the cavernous sinus (Borden type I fistulas were distributed between the transverse-sigmoid sinus $(n=10)$ and the cavernous sinus $(n=3)$.

\section{Presentation}

The main presenting symptoms were tinnitus and headache (Table 1).

Nineteen patients suffered intracranial bleeding (14 intraparenchymal, two both intraparenchymal and subarachnoid and three subarachnoid). The hemorrhage led mostly to presentation with headache $(n=9)$. Other presentations included motor deficit $(n=5)$, seizures $(n=2)$, ataxia $(n=2)$ and aphasia $(n=1)$.

Three of the $19(16 \%)$ fistulas that bled were Borden type II, the remaining $16(87 \%)$ Borden type III. In total, 
Fig. 1 Overview of patients included in study according to treatment type

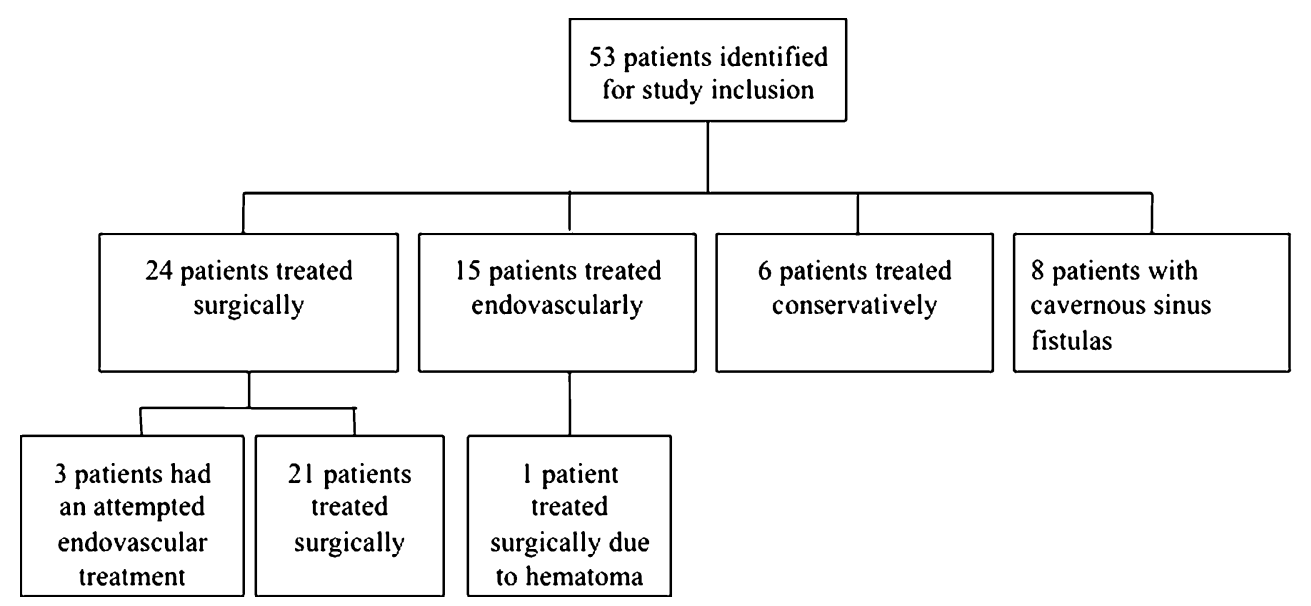

$17 \%$ of all type II fistulas presented with bleeding compared with $59 \%$ of all type III fistulas.

In eight patients with hemorrhage, the fistula was located in the superior sagittal sinus, in seven patients in the transverse-sigmoid sinus, in three patients in the posterior fossa and in one patient in the anterior cerebral fossa.

Cavernous sinus fistulas were mostly found in women (89\%). Excluding cavernous sinus fistulas - which never presented with hemorrhage - men showed a slight disposition to present with an intracranial hemorrhage, which was not significant ( $64 \%$ men vs $26 \%$ women, $p>0.05$ ). Age was not a risk factor for hemorrhagic presentation.

\section{Treatment}

Excluding the cavernous sinus fistulas where surgical treatment was not an option, we treated 24 of the patients surgically. Three patients had attempted endo-

Table 1 Overview of common signs and symptoms at clinical presentation

\begin{tabular}{lc}
\hline Signs and symptoms & Number of patients $(n)$ percentage (\%) \\
\hline Tinnitus & $13(24 \%)$ \\
Headache & $11(20 \%)$ \\
Orbital phenomena $^{a}$ & $10(19 \%)$ \\
Paresis & $5(9 \%)$ \\
Aphasia & $3(5 \%)$ \\
Ataxia & $3(5 \%)$ \\
Paresthesias & $3(5 \%)$ \\
Seizure & $3(5 \%)$ \\
Incidental & $2(4 \%)$ \\
\hline
\end{tabular}

All study patients (endovascular and surgically treated) are included in the table

${ }^{a}$ The orbital phenomena occurred with approximately equal frequency of retroorbital pulsating headache, double vision, conjunctival injection and visual blurring vascular embolization of their fistulas before their surgical treatment. All of them were Borden type II or III fistulas. Of these, 14 patients were in a clinically poor condition (Rankin grade $>2$ ).

Fifteen patients were treated endovascular (Borden type I, $n=6$; type II $n=2$, type III $n=7$ ). Six patients were managed conservatively, all of them had Borden type I fistulas.

Of the 24 surgically treated patients, we selectively disconnected the fistula in the majority of patients $(n=17)$, occluded the transverse-sigmoid sinus in five patients and both occluded and disconnected the superior sagittal sinus in two patients.

\section{Complications after surgical treatment}

Overall postoperative complications occurred in seven (29\%) surgically treated patients. One patient developed generalized cerebral swelling, two patients had a generalized seizure, one patient developed hydrocephalus, one a cerebrospinal fluid fistula, one had an epidural hematoma and one a pulmonary embolic event. Complications were independent of the type of fistula. In the majority of cases (four out of seven) patients recovered to a good outcome (mRS 0-2). Three out of seven patients with a complication had a poor outcome (mRS 3-5) but only two of the three patients worsened postoperatively. One of them had generalized cerebral swelling and the other developed a seizure disorder. The other one was in a poor condition attributable to his intracerebral bleeding. None of the postoperative complications were associated with an incomplete occlusion of the fistula. Therefore, the rate of poor outcome caused by the surgical procedure was $8 \%$ (two out of 24 patients).

One primarily endovascular treated patient had to be treated surgically because he suffered from cerebral swelling caused by his hematoma. He worsened to mRS 5. In addition, another endovascular treated patient 
suffered an infarction of the posterior circulation during coil embolization of his cavernous sinus fistula. $\mathrm{He}$ recovered to mRS 2 .

\section{Long-term outcome}

Mean follow-up time was 35 months (range 2192 months). Seven (13\%) patients were lost to follow up (four in the surgical subgroup). Figure 2 shows the outcome by mRS.

Compared with the large series of spinal DAVFs in the literature [29], our cohort of intracranial DAVFs showed that a significant number of patients (25) still suffered from residual symptoms. These included tinnitus (7), paresis (3), double vision (3) balance difficulties (5), headache (3), visual field deficits (2), tiredness (3), sensational deficits (1), aphasia (2) and seizures (1). Two patients with tinnitus had a residual Borden type I fistula, the other patients with residual symptoms had completely obliterated fistulas.

Overall, $50 \%$ showed complete remission of symptoms, $41 \%$ partial remission, $5.5 \%$ no remission and $3.7 \%$ showed a deterioration of symptoms that led to treatment of the DAVF.
Rate of events after diagnosis until treatment

During the "radiological follow-up time" [32] — the time from diagnosis of a hemorrhagic fistula until definitive treatmenttwo patients, who initially presented with bleeding, had rebleeding. The annual incidence of rebleeding in the Borden type II-III fistulas in the hemorrhagic subgroup was 23\% (two patients in 8.48 patient years). Another patient who presented with a nonhemorrhagic neurological deficit (NHND) suffered a hemorrhage 395 days after diagnosis of a Borden type II fistula in the superior sagittal sinus that was left untreated after the patient refused surgical therapy. We calculated an overall annual incidence of 30\% (three hemorrhagic events in 9.69 patient years). None of the patients with a Borden type II or III fistula who presented with pulsatile tinnitus or whose fistula was discovered incidentally had a bleeding event or developed a new NHND during the follow-up period.

\section{Incomplete treatment of DAVFs}

There were three patients that were left with incomplete occlusion of the DAVF.

One patient presented initially 2006 with aphasia and paresthesias evoked by a venous infarction. The angiogram
Fig. 2 Cumulative long-term outcome by mRS of the patients compared with their pre-operative or preinterventional status. The number of patients is shown on the $y$-axis. Seven patients were lost to follow up and are not included in the pre-operational/interventional analysis

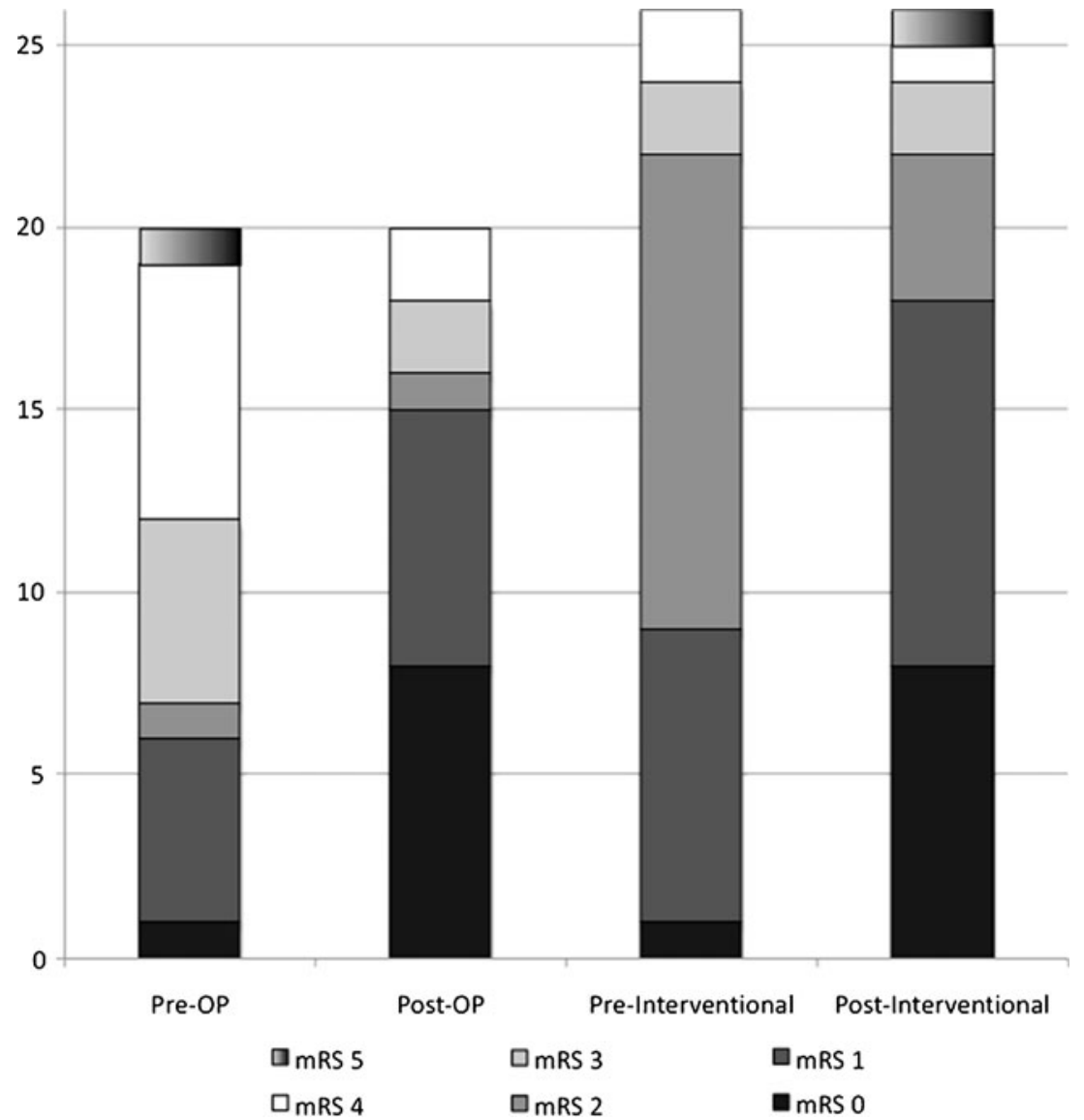


showed a Borden type III fistula to the superior sagittal sinus and a thrombosis of the superior sagittal sinus. We planned to selectively disconnect the fistula. The postoperative angiogram showed a low-flow, small, residual fistula that it was decided to keep under observation. Twelve months later she complained of a new onset of memory disturbances, which led to a new angiography that showed a recurrent Borden type III fistula.

She refused treatment but agreed to get another close follow-up angiogram in 3 months. She was mRS 0 at the time of the telephone interview.

Two patients presented with tinnitus due to a Borden type II fistula to the transverse-sigmoid sinus. We embolized the fistula and the postinterventional angiogram showed a residual Borden type I fistula. The symptoms of the patients could be reduced with regards to their intensity. A postinterventional angiogram after 12 months showed a stable Borden type I fistula. The patients were in a good condition (mRS 1) with a reduced bruit at the time of the telephone interview.

\section{Late recurrence or bleeding of occluded DAVFs}

None of the completely occluded fistulas that we managed had a rebleeding event.

Two patients had late recurrences with non-hemorrhagic symptoms where each had both surgically and endovascularly occluded DAVF. One patient presented initially with consecutive bleeding episodes that led to generalized seizures. Her fistula was Borden type III, had bilateral feeders from the occipital arteries, the tentorial arteries and was located at the transverse/sigmoid sinus junction (Fig. 3). We planned to disconnect the fistula and to occlude the trapped transverse-sigmoid sinus transition zone, but the postoperative angiogram showed incomplete disconnection with still residual filling of leptomeningeal veins. We took her back to the operating room and now also disconnected the sinus from the tentorium. The postoperative angiogram 7 days after the second operation showed complete disconnection in terms of retrograde venous drainage but still some residual filling of the distal transverse and sigmoid sinus (Fig. 4). She presented 7 months later with a new onset of seizures that initially led to her diagnosis, without evidence of rebleeding. She underwent angiography, showing a reperfusion of the fistula through bilateral feeding arteries from the occipital arteries und the ipisilateral tentorial and posterior meningeal arteries (Fig. 5). Her fistula was occluded during another surgical procedure by opening and occluding the lumen of the lateral transverse and upper sigmoid sinus with Gelfoam, paying attention not to miss a parallel venous channel [5] (Fig. 6). She developed meningitis in the late postoperative period and recovered from preoperative mRS 4 to $\mathrm{mRS} 3$.

Another patient presented 12 years after initial embolization of a transverse-sinus fistula at an outside hospital with recurrence of tinnitus. Her angiogram showed a Borden type III fistula to the superior sagittal sinus. She was successfully treated with preoperative embolization and surgical disconnection of the fistula. Unfortunately, detailed past medical records were no longer available.

\section{Discussion}

\section{Presentation of DAVFs}

Mean age at presentation was 57 years, which is very well in accordance with reports from other groups [10, 36]. Considering presentation, a variety of signs and symptoms
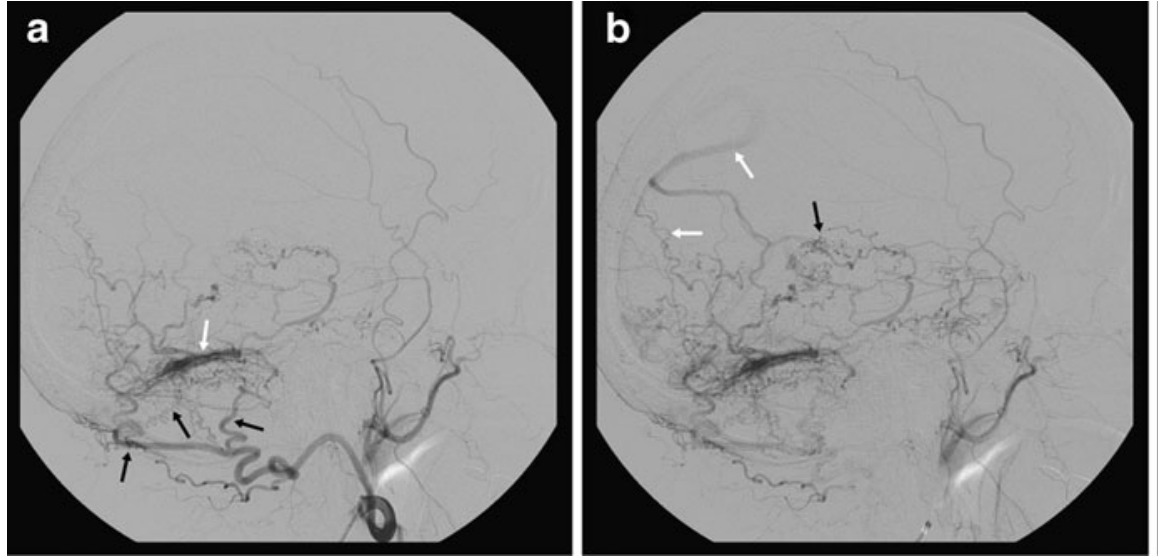

Fig. 3a-c DSA images of a patient with post-thrombotic dural AV fistula to the left transverse sinus. a Injection of the left ECA shows multiple arterial feeders, mainly from the occipital artery (black arrows) to a "trapped" segment of the transverse sinus (white arrow). b Venous outflow via multiple cortical veins (white arrows) and into the deep venous system which shows multiple small venous vessels (black arrow). c Injection of the left ICA demonstrating occlusion of the proximal left transverse sinus 
Fig. 4 a External carotid angiogram after surgical packing of the trapped sinus segment. Note persistent filling of a venous channel in the region of the left transverse sinus draining into small caliber cortical veins (arrow). b After the second surgery there is only subtle filling of the venous pouch (arrow) and no connection to cortical veins
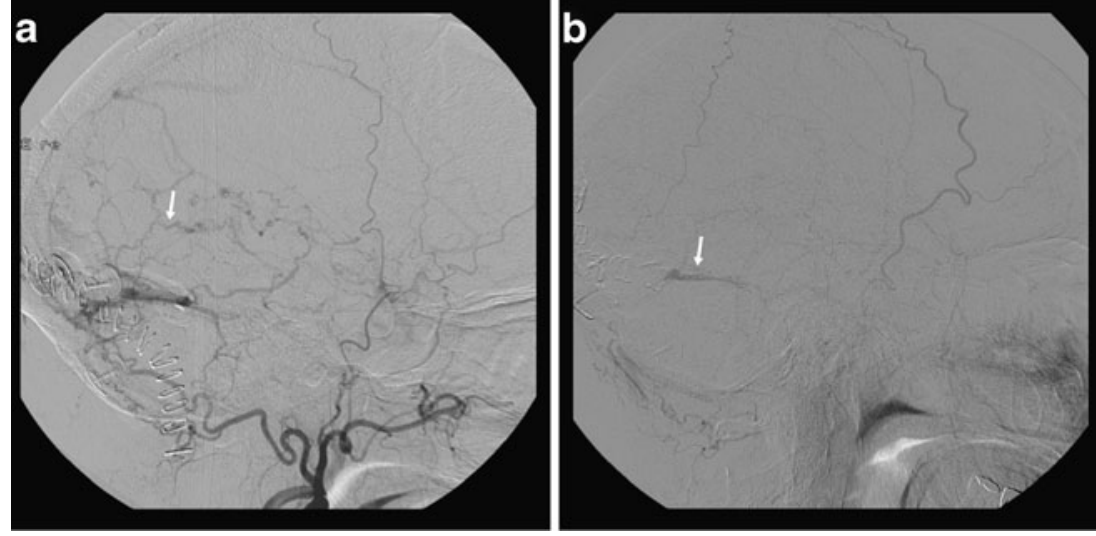

is reported in the literature that occur in very different proportions, although some percentages seem to be stable. Thirty-eight percent of our patients presented with a hemorrhage, which is higher than reported numbers of $22 \%$ [31], 18\% [18] and 16\% [11]. Most of the hemorrhagic events were intracerebral as opposed to subarachnoid. Our relation of about 3:1 (intracranial bleeding to subarachnoid bleeding) can also be found elsewhere [10]. Twenty-eight percent of these patients were over 65 years (mean age of the hemorrhagic group was 60) at the time of diagnosis. Another series of DAVFs with a higher percentage of older patients also had a higher hemorrhagic proportion [37]. A higher frequency of angiographic diagnostics in the older age group could have led to this higher number, but the exact reason will remain elusive.

Our seizure frequency of $5 \%$ is in line with published data $[31,36]$. Likewise, a significant proportion of DAVFs in this series drained to the transverse/sigmoid sinus [7]. The role of venous outflow obstruction and the revascularization of primitive fistulous tracts or even neoangiogenesis due to venous hypertension in the pathophysiology of DAVFs is widely accepted now and experimentally proven $[14,35]$. The winding course of venous outflow in the transverse and sigmoid sinus may contribute to minimal local venous hypertension with the development of fistu- lous tracts over the long term. Of note is the higher percentage of patients harboring fistulas draining to the superior sagittal sinus (20\%) in our series compared with a percentage of $5-10 \%$ that is reported in the literature [4, 31].

Risk factors for hemorrhagic events have been explored extensively in the past, with the single most important factor being retrograde leptomeningeal drainage. A recently published series of a significant number of patients added older age, male sex and location in the posterior fossa as risk factors [31]. We also had a higher percentage of male patients that suffered a hemorrhagic event, although this was not significant. We could not find any correlation with age and location. The gender-specific risk is also known from arteriovenous malformations [23] and $\mathrm{ICH}$.

\section{Indications for treatment}

Concerning bleeding risk of Borden type II and III fistulas, a prospective study by van Dijk et al. [36] reported $8.1 \%$ per year, which was also found in a retrospective Swedish study, reporting $7.8 \%$ per year [32]. In the light of a recent paper by Strom et al. [34], one must further differentiate between the bleeding risk of asymptomatic fistulas and symptomatic fistulas in close analogy to cerebral aneurysms. Asymptomatic fistulas had an annual event rate of
Fig. 5 a Common carotid injection after 6 months shows recurrence of dural AV-fistula with new feeders from the left occipital artery (black arrow) and $\mathbf{b}$ cortical venous drainage
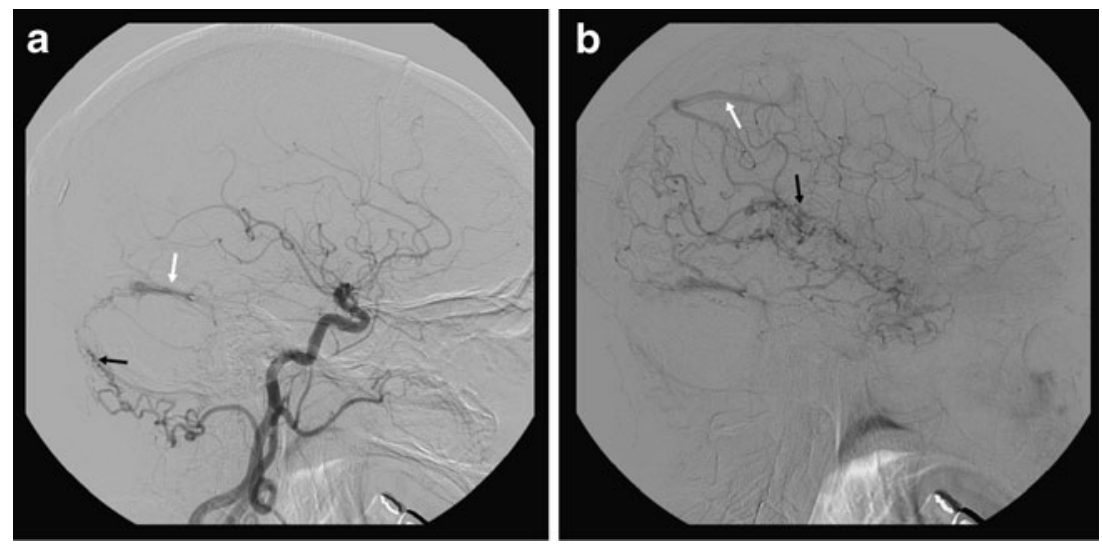


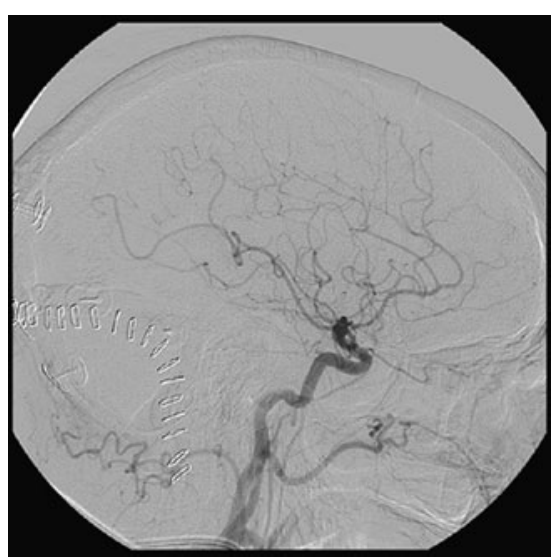

Fig. 6 Left common carotid injection after third surgery shows complete occlusion of the fistula

$1.4 \%$ versus $19.0 \%$ of symptomatic fistulas. Using the recently published approach of measuring bleeding risk with time at risk after diagnosis of the fistula [32], our overall bleeding risk would be an estimated $30 \%$ per year. This number may not represent the actual bleeding risk. None of our patients with a type II or III fistula presenting with tinnitus or incidentally had a bleeding event or experienced the development of a new NHND, which according to Strom et al. [32] shifts increases our overall estimate of bleeding risk due to bias. Furthermore, this demonstrates the limitations of the method when a short interval between diagnosis and treatment is used for calculation and therefore not enough time at risk can be assembled.

On the other hand, Duffau et al. [12] experienced a 35\% rebleeding rate in the first days (mean: 20) after the first hemorrhagic event. Contrary to this, we did not see any rebleeding event of the hemorrhagic fistulas in the first days after the diagnosis was established. It should be emphasized that the rate of bleeding as the presenting symptom is considerably higher in patients with Borden III compared with Borden II fistulas, i.e., that the direct leptomeningeal retrograde drainage translates into a higher risk of hemorrhage.

\section{Relief of symptoms}

Our mean follow-up period of 32 months lies well between previously reported 12 months [17] and 6.6 years [4]. We generally had a satisfactory outcome, with more than $85 \%$ of our patients showing mRS 0-2, Kakarla et al. [17] reported about $95 \%$ of their patients having GOS 4-5. Considering that over $50 \%$ of our patients were mRS $3-5$ preoperative/interventional, the reduction to $15 \%$ of patients being $\mathrm{mRS} 3-5$ in the long term is a satisfactory outcome.

Overall, $50 \%$ showed complete remission of symptoms, $41 \%$ partial remission, $5.5 \%$ no remission and $3.7 \%$ showed a deterioration of symptoms that led to treatment of the DAVF.

Similar results were obtained in previous studies [27]. This is comparable to spinal DAVFs [25] and probably due to the irreversible initial neuronal damage and-in the case of tinnitus - due to mechanisms still unknown to cause the symptoms primarily.

\section{Therapeutic options}

When treating a patient with a cranial DAVF, the risks of intervention must be carefully weighed against the lesion's expected clinical course based on available natural history studies. Next to a conservative approach of wait-and-see, the therapeutic armamentarium to treat cranial DAVFs today comprises microsurgical disconnection, endovascular embolization [9], selective sinus obliteration, or stereotactic radiosurgery [6]. For symptomatic Borden type II and III fistulas the high event-risk mandates immediate intervention. The therapeutic delay of stereotactic radiosurgery would be unacceptable for most patients except those with very high peri-procedural risks. Stereotactic radiosurgery may be a reasonable alternative in asymptomatic patients and those that carry a high periprocedural risk, but this has to be proven by further studies [38].

Due to technological advances, an increasing number of fistulas can and will be treated endovascularly, with excellent results in experienced hands [9, 13, 33]. Still, even in these studies, a significant number of patients will need additional surgical treatment.

Complication rates range from 5 to $10 \%[13,33]$, including permanent neurologic deficits. Outcome analysis revealed comparable results to our series [20].

When comparing traditional surgery with modern embolization techniques, it should be considered that there is significant radiation exposure during long-term embolization sessions, the danger of arterial embolization and the fact that so far only the results of the most experienced centers have been published.

Fistulas of the cavernous sinus should be reserved for endovascular management. Surgery on these lesions carries a high risk and the endovascular management has proven to have a generally good outcome $[1,2]$.

For all other locations interdisciplinary management and case-based decisions on the individual level should govern treatment. Excellent results with surgical [17] or endovascular [22] approaches have been reported. Complexity and location of the lesion and single-center experience should determine the physician's preferences of recommendations for treatment to the patient. Simple surgical disconnection is sufficient in the majority of surgical cases. 
Incomplete treatment

The goal of treatment of high-risk DAVFs must always be directed to reduction of bleeding risk and symptom relief. Apart from the landmark paper by van Dijk et al. [36], few long-term observations have been published prospectively on DAVFs with persistent cortical reflux.

Whereas downgrading of DAVFs from high risk to low risk is a well accepted treatment since Mironov's first description [24], worsening of the type of venous drainage has also been reported after incomplete treatment potentially causing catastrophic events [8]. Due to the fact that persistent cortical reflux is the major contributing factor to increased bleeding risk, incomplete treatment should be corrected by a subsequent second procedure. Spontaneous resolution of higher grade DAVFs within 2-10 months has been reported, although the sizes of DAVFs were not specified [28, 30].

\section{Late recurrence}

We saw two patients who had a late recurrence of their primarily endovascular (one case) and surgically (one case) treated fistula. According to one hypothesis, primordial arterio-venous channels that persist after embryologic closure that are reperfused due to a shift in pressure gradients after an increase in venous intravascular pressure $[16,35]$. The fistula that was treated surgically was disconnected and the transverse and sigmoid sinus trapped. The postoperative angiogram showed complete disconnection but residual filling of the trapped sinus. This may have indicated that flow dynamics were not completely changed and may have facilitated opening of new fistula channels. The phenomenon of reperfusion is only scarcely reported in the literature $[15$, $19,28]$.

\section{Reducing complications in surgery}

Although our peri-operative complication rate of $29 \%$ is well in line with previously reported rates of $32 \%$ [17], it has to be emphasized, however, that the rate of clinically significant complications was less than $10 \%$ (two out of 24 patients deteriorated to a Rankin grade $>2$ after surgery). Careful close pre- and postoperative monitoring and a judicious allocation to the different treatment modalities are mandatory for these patients. Redirecting cerebral blood flow often carries an inherent risk of experiencing unexpected complications unrelated to treatment modalities. Very low surgical complication rates with complications having no influence on postoperative outcome have also been reported, but should be interpreted cautiously [10].

\section{Conclusion}

Here we present our 8-year experience of the management of DAVFs. The major determinants for hemorrhagic eventsleptomeningeal venous drainage and gender-were also found in our cohort. We showed that, in general, DAVFs can be managed, leading to a very satisfactory outcome; however, half of the patients do not show complete remission of symptoms. Age and the occurrence of perioperative complication were the most important determinants of outcome. The phenomenon of reperfusion should be considered in the follow-up of these patients.

Acknowledgements We thank Marina Eberhardt for her valuable assistance with figure design. Editing support was provided for the final manuscript by Susan Wieting, University Bern, Neurosurgery Department.

Conflicts of interest None.

\section{References}

1. Bink A, Berkefeld J, Kraus L, Senft C, Ziemann U, de Rochemont $\mathrm{R}$ du Mesnil (2010) Long-term outcome in patients treated for benign dural arteriovenous fistulas of the posterior fossa. Neuroradiology. doi:10.1007/s00234-010-0754-x

2. Bink A, Goller K, Luchtenberg M, Neumann-Haefelin T, Dutzmann S, Zanella F, Berkefeld J, de Rochemont R du Mesnil (2010) Long-term outcome after coil embolization of cavernous sinus arteriovenous fistulas. AJNR Am J Neuroradiol 31:12161221

3. Borden JA, Wu JK, Shucart WA (1995) A proposed classification for spinal and cranial dural arteriovenous fistulous malformations and implications for treatment. J Neurosurg 82:166-179

4. Brown RD Jr, Wiebers DO, Nichols DA (1994) Intracranial dural arteriovenous fistulae: angiographic predictors of intracranial hemorrhage and clinical outcome in nonsurgical patients. J Neurosurg 81:531-538

5. Caragine LP, Halbach VV, Dowd CF, Ng PP, Higashida RT (2003) Parallel venous channel as the recipient pouch in transverse/ sigmoid sinus dural fistulae. Neurosurgery 53:1261-1266

6. Chandler HC Jr, Friedman WA (1993) Successful radiosurgical treatment of a dural arteriovenous malformation: case report. Neurosurgery 33:139-141

7. Cognard C, Gobin YP, Pierot L, Bailly AL, Houdart E, Casasco A, Chiras J, Merland JJ (1995) Cerebral dural arteriovenous fistulas: clinical and angiographic correlation with a revised classification of venous drainage. Radiology 194:671-680

8. Cognard C, Houdart E, Casasco A, Gabrillargues J, Chiras J, Merland JJ (1997) Long-term changes in intracranial dural arteriovenous fistulae leading to worsening in the type of venous drainage. Neuroradiology 39:59-66

9. Cognard C, Januel AC, Silva NA Jr, Tall P (2008) Endovascular treatment of intracranial dural arteriovenous fistulas with cortical venous drainage: new management using Onyx. AJNR Am J Neuroradiol 29:235-241

10. Collice M, D'Aliberti G, Arena O, Solaini C, Fontana RA, Talamonti G (2000) Surgical treatment of intracranial dural arteriovenous fistulae: role of venous drainage. Neurosurgery 47:56-66 
11. Davies MA, TerBrugge K, Willinsky R, Coyne T, Saleh J, Wallace MC (1996) The validity of classification for the clinical presentation of intracranial dural arteriovenous fistulas. J Neurosurg 85:830-837

12. Duffau H, Lopes M, Janosevic V, Sichez JP, Faillot T, Capelle L, Ismail M, Bitar A, Arthuis F, Fohanno D (1999) Early rebleeding from intracranial dural arteriovenous fistulas: report of 20 cases and review of the literature. J Neurosurg 90:78-84

13. Guedin P, Gaillard S, Boulin A, Condette-Auliac S, Bourdain F, Guieu S, Dupuy M, Rodesch G (2010) Therapeutic management of intracranial dural arteriovenous shunts with leptomeningeal venous drainage: report of 53 consecutive patients with emphasis on transarterial embolization with acrylic glue. J Neurosurg 112:603-610

14. Hamada $\mathrm{Y}$, Goto $\mathrm{K}$, Inoue $\mathrm{T}$, Iwaki $\mathrm{T}$, Matsuno $\mathrm{H}$, Suzuki $\mathrm{S}$, Matsushima T, Fukui M, Miyake E (1997) Histopathological aspects of dural arteriovenous fistulas in the transverse-sigmoid sinus region in nine patients. Neurosurgery 40:452-456

15. Hiu T, Horie N, Hayashi K, Kitagawa N, Morikawa M, Kawakubo J, Tsutsumi K, Suyama K, Nagata I (2008) Recurrence of the cavernous sinus dural arteriovenous fistula at adjacent sinuses following repeated transvenous embolizations: case report and literature review. Radiat Med 26:431-437

16. Ishikawa T, Sato S, Sasaki T, Matsumoto M, Suzuki K, Itakura T, Kodama N, Hiraiwa K (2007) Histologic study of arteriovenous shunts in the normal dura mater adjacent to the transverse sinus. Surg Neurol 68:272-276

17. Kakarla UK, Deshmukh VR, Zabramski JM, Albuquerque FC, McDougall CG, Spetzler RF (2007) Surgical treatment of highrisk intracranial dural arteriovenous fistulae: clinical outcomes and avoidance of complications. Neurosurgery 61:447-457

18. Kim MS, Han DH, Kwon OK, Oh CW, Han MH (2002) Clinical characteristics of dural arteriovenous fistula. J Clin Neurosci 9:147-155

19. Kiyosue H, Tanoue S, Okahara M, Yamashita M, Nagatomi H, Mori H (2002) Recurrence of dural arteriovenous fistula in another location after selective transvenous coil embolization: report of two cases. AJNR Am J Neuroradiol 23:689-692

20. Lee CW, Huang A, Wang YH, Yang CY, Chen YF, Liu HM (2010) Intracranial dural arteriovenous fistulas: diagnosis and evaluation with 64-detector row CT angiography. Radiology 256:219-228

21. Lindsay Wilson AH (2002) Structured Interview for the Modified Rankin Scale Questionnaire Guidelines, http://www.psychology. stir.ac.uk/staff/lwilson/documents/MRS_Structured_Interview.pdf. Accessed January 2009

22. Macdonald JH, Millar JS, Barker CS (2009) Endovascular treatment of cranial dural arteriovenous fistulae: a single-centre, 14-year experience and the impact of Onyx on local practise. Neuroradiology 52:387-95

23. Mast H, Young WL, Koennecke HC, Sciacca RR, Osipov A, PileSpellman J, Hacein-Bey L, Duong H, Stein BM, Mohr JP (1997) Risk of spontaneous haemorrhage after diagnosis of cerebral arteriovenous malformation. Lancet 350:1065-1068
24. Mironov A (1998) Selective transvenous embolization of dural fistulas without occlusion of the dural sinus. AJNR Am J Neuroradiol 19:389-391

25. Narvid J, Hetts SW, Larsen D, Neuhaus J, Singh TP, McSwain H, Lawton MT, Dowd CF, Higashida RT, Halbach VV (2008) Spinal dural arteriovenous fistulae: clinical features and long-term results. Neurosurgery 62:159-166

26. Nelson PK, Russell SM, Woo HH, Alastra AJ, Vidovich DV (2003) Use of a wedged microcatheter for curative transarterial embolization of complex intracranial dural arteriovenous fistulas: indications, endovascular technique, and outcome in 21 patients. $\mathrm{J}$ Neurosurg 98:498-506

27. Olteanu-Nerbe V, Uhl E, Steiger HJ, Yousry T, Reulen HJ (1997) Dural arteriovenous fistulas including the transverse and sigmoid sinuses: results of treatment in 30 cases. Acta Neurochir 139:307318

28. Pan HC, Sheehan J, Huang CF, Yang DY (2007) Two consecutive dural arteriovenous fistulae in a child: a case report of successful treatment with gamma knife radiosurgery. Childs Nerv Syst 23:1185-1190

29. Saladino A, Atkinson JL, Rabinstein AA, Piepgras DG, Marsh WR, Krauss WE, Kaufmann TJ, Lanzino G (2010) Surgical treatment of spinal dural arteriovenous fistulae: a consecutive series of 154 patients. Neurosurgery 67:1350-1357

30. Satomi J, van Dijk JM, Terbrugge KG, Willinsky RA, Wallace MC (2002) Benign cranial dural arteriovenous fistulas: outcome of conservative management based on the natural history of the lesion. J Neurosurg 97:767-770

31. Singh V, Smith WS, Lawton MT, Halbach VV, Young WL (2008) Risk factors for hemorrhagic presentation in patients with dural arteriovenous fistulae. Neurosurgery 62:628-635

32. Soderman M, Pavic L, Edner G, Holmin S, Andersson T (2008) Natural history of dural arteriovenous shunts. Stroke 39:1735-1739

33. Stiefel MF, Albuquerque FC, Park MS, Dashti SR, McDougall CG (2009) Endovascular treatment of intracranial dural arteriovenous fistulae using Onyx: a case series. Neurosurgery 65:132-139

34. Strom JA, Towne JB, Quebbeman EJ, Bernhard VM (1984) Autotransfusion in complex abdominal aneurysms. Surg Gynecol Obstet 159:59-62

35. Terada T, Higashida RT, Halbach VV, Dowd CF, Tsuura M, Komai N, Wilson CB, Hieshima GB (1994) Development of acquired arteriovenous fistulas in rats due to venous hypertension. J Neurosurg 80:884-889

36. van Dijk JM, terBrugge KG, Willinsky RA, Wallace MC (2002) Clinical course of cranial dural arteriovenous fistulas with longterm persistent cortical venous reflux. Stroke 33:1233-1236

37. Willinsky R, Goyal M, terBrugge K, Montanera W (1999) Tortuous, engorged pial veins in intracranial dural arteriovenous fistulas: correlations with presentation, location, and MR findings in 122 patients. AJNR Am J Neuroradiol 20:1031-1036

38. Yang HC, Kano H, Kondziolka D, Niranjan A, Flickinger JC, Horowitz MB, Lunsford LD (2010) Stereotactic radiosurgery with or without embolization for intracranial dural arteriovenous fistulas. Neurosurgery 67:1276-1283 